\title{
Contribution of the new 2012 EULAR/ACR classification criteria for the diagnosis of polymyalgia rheumatica
}

\author{
F. Muratore ${ }^{1,2}$, C. Salvarani ${ }^{1,2}$, P. Macchioni ${ }^{1}$ \\ 'Division of Rheumatology, Azienda USL-IRCCS di Reggio Emilia; \\ ${ }^{2}$ University of Modena and Reggio Emilia, Italy
}

\section{SUMMARY}

Polymyalgia rheumatica (PMR) is one of the most common rheumatic inflammatory disorders in people aged over 50. It is characterized by aching and prolonged morning stiffness in the shoulder and pelvic girdles and neck. To date there are no specific diagnostic tests, and in clinical practice the diagnosis of PMR remains based on its characteristic clinical manifestations, laboratory evidence of systemic inflammation, rapid response to low doses of glucocorticoids and exclusion of other disorders that may present with proximal pain and stiffness. For classification purposes, several criteria have been proposed over time based on retrospective clinical series, but none have been validated and received universal acceptance. Recently, an international collaborative initiative between the EULAR and the ACR was undertaken to develop new polymyalgia rheumatica classification criteria. In this review, the provisional 2012 EULAR/ACR classification criteria will be presented and their contribution for the diagnosis of polymyalgia rheumatica will be discussed.

Key words: Polymyalgia rheumatica; Classification criteria.

Reumatismo, 2018; 70 (1): 18-22

\section{THE NEW 2012 EULAR/ACR CLASSIFICATION CRITERIA}

Pinst olymyalgia rheumatica (PMR) is an inflammatory disorder of unknown cause characterized by aching and prolonged morning stiffness in the shoulder, pelvic girdles and neck, affecting people aged over 50. It is one of the most common inflammatory rheumatic conditions in Caucasian people aged over 50 and its incidence increases with advancing age, peaking between 70 and 80 years of age. A well-known association exists between PMR and giant cell arteritis (GCA), a large vessel vasculitis that affects the aorta and its branches (1). Controversy exists as to whether PMR represents a single entity disease or is an umbrella term that comprises a clinical presentation common to a range of related conditions (polymyalgic syndrome) (2). To date there are no specific diagnostic tests, and in clinical practice the diagnosis of PMR remains based on its characteristic clinical manifestations, laboratory evidence of systemic inflammation, rapid response to low doses of glucocorticoids (GCs) and exclusion of other disorders that may present with polymyalgic syndrome (proximal pain and stiffness). Ultrasonographic findings of peri-articular inflammation lend further support to the diagnosis of PMR (3).

Many features of PMR may predispose the unwary clinician to diagnostic error. The main symptoms of PMR, such as proximal pain and stiffness syndrome, systemic symptoms, distal musculoskeletal manifestation and evidence of systemic inflammation, can occur in many other illnesses. Furthermore, the use of a response to GC therapy to confirm the diagnosis of PMR has several limitations, since GCs are potent anti-inflammatory agents that can mask symptoms of other serious conditions, especially if used in high doses and for protracted periods. Early differentiation between PMR and late onset rheumatoid 
arthritis (LORA) with PMR-like onset can be difficult because these conditions may have similar clinical presentation (4). Follow-up is often needed to establish the correct diagnosis, and in up to $30 \%$ of patients initially diagnosed as PMR, the disease is eventually reclassified as LORA (5).

The International PMR Classification Criteria Group endorsed by ACR and EULAR has agreed an approach for the polymyalgic syndrome that sees the diagnosis of PMR as a step-wise process $(6,7)$ :

1. evaluate for core inclusion criteria: age over 50; bilateral shoulder and/or pelvic girdle aching; morning stiffness duration longer than $45 \mathrm{~min}$; evidence of an acute-phase response;

2. evaluate for exclusion criteria: exclude mimicking conditions that cannot coexist with and rule out the diagnosis of PMR;

3. evaluate a standardized response to GCs $(15 \mathrm{mg} /$ day of prednisolone or its equivalent): response is defined as a patient-reported global improvement of $70 \%$ within a week of commencing GCs and normalization of inflammatory markers within 4 weeks. A lesser response should encourage the search for an alternative condition;

4. confirmation of the diagnosis on followup: evaluate response to GCs and exclude mimicking conditions during the follow-up.

For classification purposes, several criteria have been proposed over time based on retrospective clinical series (Table I) (811). However, none of these criteria have been validated and received universal acceptance. Most of these classification criteria include an age cutoff, the presence of shoulder and hip girdle pain and morning stiffness, duration of symptoms lasting more than 2-4 weeks, elevated markers of inflammation and the exclusion of other diagnoses. Furthermore, some of these classification criteria include a rapid response to GC therapy $(9,11)$. However, the use of a rapid and dramatic response to GC therapy to confirm the diagnosis of PMR has limitations. Several recent studies have indeed shown that about one third of patients with PMR treated with GCs according to a standard protocol do not have a complete response even after 3 to 4 weeks of treatment (12-14). One of the major unresolved issues is the lack of laboratory and/or imaging tests that allow early differentiation between PMR and other inflammatory rheumatic diseases, particularly from RA. Regarding this, preliminary studies have shown that musculoskeletal ultrasound of the shoulders and hips, and antibodies to cyclic citrullinated peptides, can differentiate PMR from RA with high specificity and acceptable sensitivity (15-18).

The lack of standardized classification criteria has been a major factor hampering the development of rational therapeu-

Table I - Proposed diagnostic criteria for polymyalgia rheumatica.

\section{Chuang et al. (8)}

The presence of all these criteria defines PMR diagnosis:

1. Patients 50 years or older

2. Bilateral aching and stiffness persisting for one month or more involving two of the following areas: neck or torso, shoulders or proximal regions of the arms, and hips or proximal aspects of the thighs

3. Erythrocyte sedimentation rate greater than $40 \mathrm{~mm} / 1^{\text {st }}$ hour

4. Exclusion of other diagnoses except giant cell arteritis

\section{Healey (9)}

The diagnosis of PMR is made if all these criteria are satisfied:

1. Persistent pain (for at least one month) involving two of the following areas: neck, shoulders, and pelvic girdle

2. Morning stiffness lasting more than $1 \mathrm{~h}$

3. Rapid response to prednisone ( $20 \mathrm{mg} /$ day or less)

4. Absence of other diseases capable of causing the musculoskeletal symptoms

5. Age over 50 years

6. Erythrocyte sedimentation rate greater than $40 \mathrm{~mm} / 1^{\text {st }}$ hour

\section{Bird et al. (10)}

A diagnosis of probable PMR is made if any 3 or more of these criteria are fulfilled:

1. Bilateral shoulder pain and/or stiffness

2. Onset of illness within two weeks

3. Initial erythrocyte sedimentation rate higher than $40 \mathrm{~mm} / 1$ st hour

4. Morning stiffness exceeding $1 \mathrm{~h}$

5. Age over 65 years

6. Depression and/or loss of weight

7. Bilateral upper arm tenderness

The presence of any 3 or more criteria yields a sensitivity of $92 \%$ and a specificity of $80 \%$

\section{Jones and Hazleman (11)}

Diagnosis of polymyalgia rheumatica requires presence of all the following:

1. Shoulder and pelvic girdle pain, mainly muscular but without muscle weakness

2. Duration of symptoms at least 2 months

3. Morning stiffness

4. ESR $>30 \mathrm{~mm} / \mathrm{h}$ or C-reactive protein $>57.14 \mathrm{nmol} / \mathrm{L}(6 \mathrm{mg} / \mathrm{L})$

5. Absence of rheumatoid factor, inflammatory arthritis, and malignant disease

6. Absence of objective signs of muscle disease

7. Prompt and pronounced response to glucocorticoids 
tic approaches and causing difficulties in evaluating patients in clinical studies. To overcome these limitations, an international collaborative initiative between the EULAR and the ACR was undertaken to develop new PMR classification criteria $(12,13)$. These criteria were generated by a prospective evaluation of a cohort of 125 patients with new-onset PMR and 169 subjects aged over 50 with new-onset bilateral shoulder pain secondary to conditions potentially mimicking PMR. Musculoskeletal ultrasound of the shoulders and hips was done in both groups at baseline and at 26 weeks. The investigators then developed a scoring algorithm (Table II) $(12,13)$. In patients aged 50 years or older presenting with bilateral shoulder aching and raised inflammatory markers (ESR and/or CRP), a score of clinical criteria $\geq 4$ had a sensitivity of $68 \%$ and specificity of $78 \%$ for discriminating polymyalgia rheumatica from control patients. The specificity was higher $(88 \%)$ for discriminating shoulder conditions from PMR and lower $(65 \%)$ for discriminating RA from PMR. The use of ultrasound was discretionary; when ultrasonography findings consistent with PMR were considered, sensitivity decreased to $66 \%$, but specificity increased to $81 \%$. Again, the specificity was higher (89\%) for discriminating shoulder conditions from PMR and lower (70\%) for discriminating RA from PMR $(12,13)$. Therefore, ultrasound findings were useful in discriminat- ing PMR from shoulder conditions, but less so in discriminating PMR from RA. However, ultrasound findings had no effect on the sensitivity of the novel EULAR/ACR criteria for $\operatorname{PMR}(12,13,19)$.

To date only two studies have assessed the performance of the new 2012 provisional EULAR/ACR PMR clinical classification criteria in discriminating PMR from other mimicking conditions compared with the previous published classification criteria, reporting conflicting results $(20,21)$.

In a single-center retrospective study (20), our group compared the performance of the published classification/diagnostic criteria for PMR, including the new EULAR/ ACR provisional criteria, in $136 \mathrm{PMR}$ patients and 149 controls (including 94 with rheumatoid arthritis), all prospectively followed up according to a standardized protocol that included ultrasound examination of shoulders and hips. The most sensitive criteria were the new EULAR/ACR provisional classification criteria (92.6\%). Adding ultrasound, specificity increased from $81.5 \%$ to $91.3 \%$ when all comparators were considered and from $79.7 \%$ to $89.9 \%$ when only rheumatoid arthritis patients were considered. Bird criteria had a sensitivity of $89.2 \%$ but the lowest specificity $(40.2 \%$ in total cases and $72.5 \%$ in RA). Jones and Nobunaga criteria were the most specific criteria $(96.7 \%$ and $97.8 \%$ in total cases and $98.6 \%$ and $99.5 \%$ in RA) but the less sensitive $(63.1 \%$ and $58.2 \%)$.

Table II - European League Against Rheumatism and American College of Rheumatology provisional criteria for classification of polymyalgia rheumatica.

\begin{tabular}{|l|l|}
\hline \multicolumn{2}{|l|}{ Required criteria: age 50 years or older, bilateral shoulder aching and abnormal C-reactive protein and/or } \\
erythrocyte sedimentation rate & \\
\hline Clinical criteria for scoring algorithm:* & 2 points \\
1. Morning stiffness lasting more than 45 min & 1 point \\
2. Hip pain or restricted range of motion & 2 points \\
3. Absence of rheumatoid factor and antibody to cyclic citrullinated peptide & 1 point \\
4. Absence of other joint involvement & \\
\hline $\begin{array}{l}\text { Ultrasound criteria for scoring algorithm:* } \\
\text { 5a. At least one shoulder with subdeltoid bursitis, biceps tenosynovitis, or glenohumeral synovitis; } \\
\text { and at least one hip with synovitis or trochanteric bursitis }\end{array}$ & 1 point \\
5b. Both shoulders with subdeltoid bursitis, biceps tenosynovitis, or glenohumeral synovitis & 1 point \\
\hline
\end{tabular}

*With only clinical criteria, a score of $\geq 4$ had a sensitivity of $68 \%$ and specificity of $78 \%$ for discriminating polymyalgia rheumatica from comparison patients. With a combination of clinical criteria and ultrasound criteria, a score of $\geq 5$ had a sensitivity of $66 \%$ and specificity of $81 \%$ for discriminating patients with the disorder from comparison patients. 
Healey and Chuang criteria had similar intermediate sensitivity $(80.3 \%$ and $77 \%)$ and specificity $(81.5 \%$ in total cases and $78.3 \%$ in RA). A more recent prospective multicenter study assessed the performance of the published classification/diagnostic criteria for PMR, including the new EULAR/ACR provisional criteria, in 275 patients with new-onset bilateral shoulder pain (21). At baseline evaluation, 145 patients $(52.7 \%)$ were diagnosed as PMR and $130(47.3 \%)$ as non-PMR. At the end of the 1-year follow-up, 133 patients $(48.4 \%)$ were diagnosed with PMR and 142 (51.6\%) were diagnosed as non-PMR (including 69 with RA). The 2012 EULAR/ACR clinical criteria for PMR had a sensitivity of $89.5 \%$ and a specificity of $57.7 \%$ when tested against all non-PMR cases. Compared with these new classification criteria, only the Bird criteria had higher sensitivity $(94 \%)$, with lower specificity $(50 \%)$. However, the specificities of the other criteria sets were significantly higher than the new 2012 EULAR/ACR clinical criteria, ranging from $83 \%-93 \%$. The Jones criteria and the Chuang criteria had the highest specificities $(93.7 \%$ and $88 \%$, respectively). Surprisingly, when the new 2012 EULAR/ACR clinical criteria for PMR were tested against the 69 RA cases, specificity increased to $66.7 \%$. Shoulder and hip ultrasound were performed only in a subgroup of 48 patients. With the use of the ultrasonography criteria, the sensitivity of the 2012 EULAR/ACR criteria increased to $91.3 \%$ but the specificity decreased to $52 \%$ for discriminating non-PMR conditions from PMR and $53.8 \%$ for discriminating RA from PMR. This unexpected finding of Ozen's study is in disagreement with both the original study and the study by Macchioni, et al, which showed more specificity than sensitivity and a better performance when the criteria were used to discriminate between PMR and noninflammatory shoulder diseases rather than between PMR and RA. This observation is surprising, because late-onset RA is unanimously considered the most challenging differential diagnosis. It is difficult to find substantial methodological differences be- tween the three studies that could explain the different results obtained. Differences in the study design and in the criteria used to include patients and controls and the clinician's ability to make a diagnosis and perform ultrasonography could, at least in part, explain the different results obtained (22). Prospective, multicenter studies recruiting a larger number of controls with arthritis and other conditions mimicking PMR are required.

In conclusion, PMR is one of the most common inflammatory conditions in patients aged over 50. Various criteria have been proposed for the classification of the disease, but in clinical practice the diagnosis remains only clinical. Imaging modalities may support the clinical diagnosis of PMR, and US has been shown to slightly improve the specificity of the diagnosis. The ACR/EULAR criteria seem to constitute progress in the classification of PMR, although they are provisional and need to be validated in further prospective studies and different settings. Furthermore, these criteria are meant for classification and not for diagnostic purposes, have not been tested as diagnostic criteria, and can only be applied to patients in whom an alternative diagnosis responsible for the shoulder pain has already been excluded.

Conflict of interest: the authors declare no conflict of interest.

\section{REFERENCES}

1. Salvarani C, Cantini F, Boiardi L, Hunder GG. Polymyalgia rheumatica and giant cell arteritis. N Engl J Med. 2002; 347: 261-71.

2. Muratore F, Pazzola G, Pipitone N, Salvarani C. Recent advances in the diagnosis and treatment of polymyalgia rheumatica. Expert Rev Clin Immunol. 2016; 12: 1037-45.

3. Macchioni P, Catanoso MG, Pipitone N, et al. Longitudinal examination with shoulder ultrasound of patients with polymyalgia rheumatica. Rheumatology (Oxford). 2009; 48: 1566-9.

4. Caporali R, Montecucco C, Epis O, et al. Presenting features of polymyalgia rheumatica and rheumatoid arthritis with PMR-like onset: a prospective study. Ann Rheum Dis. 2001; 60: 1021-4. 
5. Kermani TA, Warrington KJ. Polymyalgia rheumatica. Lancet. 2013; 381: 63-72.

6. Dasgupta B, Borg FA, Hassan N, et al. BSR and BHPR guidelines for the management of polymyalgia rheumatica. Rheumatology (Oxford). 2010; 49: 186-90.

7. Dasgupta B, Hutchings A, Matteson EL. Polymyalgia rheumatica: the mess we are now in and what we need to do about it. Arthritis Rheum. 2006; 55: 518-20.

8. Chuang TY, Hunder GG, Ilstrup DM, Kurland LT. Polymyalgia rheumatica: a 10-year epidemiologic and clinical study. Ann Intern Med. 1982; 97: 672-80.

9. Healey LA. Long-term follow-up of polymyalgia rheumatica: evidence for synovitis. Semin Arthritis Rheum. 1984; 13: 322-8.

10. Bird HA, Esselinckx W, Dixon AS, et al. An evaluation of criteria for polymyalgia rheumatica. Ann Rheum Dis. 1979; 38: 434-9.

11. Jones JG, Hazleman BL. Prognosis and management of polymyalgia rheumatica. Ann Rheum Dis. 1981; 40: 1-5.

12. Dasgupta B, Cimmino MA, Kremers HM, et al. 2012 provisional classification criteria for polymyalgia rheumatica: a European League Against Rheumatism/American College of Rheumatology collaborative initiative. Arthritis Rheum. 2012; 64: 943-54.

13. Dasgupta B, Cimmino MA, Maradit-Kremers $\mathrm{H}$, et al. 2012 provisional classification criteria for polymyalgia rheumatica: a European League Against Rheumatism/American College of Rheumatology collaborative initiative. Ann Rheum Dis. 2012; 71: 484-92.

14. Hutchings A, Hollywood J, Lamping DL, et al. Clinical outcomes, quality of life, and diagnostic uncertainty in the first year of polymyalgia rheumatica. Arthritis Rheum. 2007; 57: 803-09.

15. Cantini F, Salvarani C, Olivieri I, et al. Shoul- der ultrasonography in the diagnosis of polymyalgia rheumatica: a case-control study. J Rheumatol. 2001; 28: 1049-55.

16. Scheel AK, Matteson EL, Dasgupta B, et al. Reliability exercise for the polymyalgia rheumatica classification criteria study: the oranjewoud ultrasound substudy. Int J Rheumatol. 2009; 2009: 738931.

17. Lopez-Hoyos M, Ruiz de Alegria C, Blanco $\mathrm{R}$, et al. Clinical utility of anti-CCP antibodies in the differential diagnosis of elderly-onset rheumatoid arthritis and polymyalgia rheumatica. Rheumatology (Oxford). 2004; 43: 655-7.

18. Dasgupta B, Hutchings A, Hollywood J, Nutter L. Autoantibodies to cyclic citrullinated peptide in a PMR inception cohort from The PMR Outcomes Study. Ann Rheum Dis. 2008; 67: 903-4.

19. Weigand S, Ehrenstein B, Fleck M, Hartung W. Joint involvement in patients with early polymyalgia rheumatica using high-resolution ultrasound and its contribution to the EULAR/ACR 2012 classification criteria for polymyalgia rheumatica. J Rheumatol. 2014; 41: 730-4

20. Macchioni P, Boiardi L, Catanoso M, et al. Performance of the new 2012 EULAR/ACR classification criteria for polymyalgia rheumatica: comparison with the previous criteria in a single-centre study. Ann Rheum Dis. 2014; 73: 1190-3.

21. Ozen G, Inanc N, Unal AU, et al. Assessment of the new 2012 EULAR/ACR clinical classification criteria for polymyalgia rheumatica: a prospective multicenter study. J Rheumatol. 2016; 43: 893-900.

22. Camellino D, Cimmino MA. Are the new ACR/EULAR criteria the ultimate answer for polymyalgia rheumatica classification? J Rheumatol. 2016; 43: 836-8. 